\title{
Hohes Suizidrisiko direkt nach der Entlassung
}

Fragestellung: Suizidhäufigkeiten nach der Entlassung aus psychiatrischen Einrichtungen sollten quantifiziert und Einflussfaktoren identifiziert werden.

Hintergrund: Nahezu alle psychischen Störungen weisen eine erhöhte Suizidmortalität auf. Patienten, die kürzlich aus stationärer psychiatrischer Behandlung entlassen wurden, scheinen ein besonders hohes Suizidrisiko zu haben. Die Kenntnis dieser Phasen mit hohem Risiko sowie entsprechender Einflussfaktoren erlaubt es, Schlüsse für die Prävention zu ziehen.

Patienten und Methodik: In den Datenbanken EMBASE, MEDLINE und PsycINFO wurden Studien identifiziert, die Angaben zu Suizidraten nach der Entlassung aus psychiatrischen Kliniken sowie zu Personenjahren bis zum Suizid enthielten, und im Rahmen einer Metaanalyse ausge-

Chung DT, Ryan CJ, HadziPavlovic $D$ et al. Suicide rates after discharge from psychiatric facilities: A systematic review and metaanalysis. JAMA

Psychiatry 2017; 74: 694-702 wertet.

Ergebnisse: Insgesamt 100 Studien mit zusammen 183 Patientenstichproben wurden analysiert. Die Auswertung umfasste 17.857 Suizide über
4.725.445 Personenjahre. Die mittlere gepoolte Suizidrate über alle Studien nach Entlassung betrug 484/100.000 Personenjahre (95\%-Konfidenzintervall [KI] 422 bis 555 Suizide pro 100.000 Personenjahre). Die Suizidrate war am höchsten im Zeitraum bis zu drei Monate nach der Entlassung mit 1.132/100.000 Personenjahre (95\%-KI 874 - 1.467) und für Patienten mit Suizidgedanken oder -versuchen zum Aufnahmezeitpunkt ( $\mathrm{n}=2.078$; 95\%-KI 1.512-2.856). Für den Zeitraum von drei Monaten bis zu einem Jahr nach Entlassung ergaben sich 654 Suizide, für ein bis fünf Jahre 494 und für fünf bis zehn Jahren 366 Suizide/100.000 Personenjahre. Bei einem Follow-up-Intervall von länger als zehn Jahren traten 277 Suizide pro 100.000 Personenjahre auf. Stichproben mit Patienten, die aufgrund von Suizidalität aufgenommen wurden, wiesen eine über das Vierfache erhöhte poststationäre Suizidrate auf (2.078/100.000 vs. 452/100.000 Personenjahre; $\mathrm{p}<0,001)$. In jüngeren Stichproben waren die Suizidraten höher als in älteren (95\%-KI 0,008-0,031).

Schlussfolgerungen: Bis zu drei Monate nach der Entlassung besteht bei psychiatrischen Patienten eine besonders hohe Suizidvulnerabilität. Die Suizidraten bleiben auch Monate bis Jahre danach sehr hoch. Zudem stellt Suizidalität bei Aufnahme einen ernst zu nehmenden Risikofaktor dar.

\section{- Kommentar von Christian Lange-Asschenfeldt, Düsseldorf}

\section{Patienten nach der Klinikentlassung nicht sich selbst überlassen}

Die Autoren konnten weltweit über die unterschiedlichsten Versorgungssettings hinweg zeigen, dass die poststationäre Suizidrate in den vergangenen Jahrzehnten trotz aller Verbesserung der Versorgungssysteme, Fortschritte in der psychosozialen Integration und Destigmatisierung psychisch Kranker sowie hinsichtlich medikamentöser und psychotherapeutischer Behandlungsstrategien nicht abgenommen hat, sondern im Gegenteil noch angestiegen ist. Dem entsprechen auch neuere Befunde zum Anstieg der Suizidrate bei stationär behandelten Patienten [1]. Die vorliegende Studie zeigt vor allem, dass die ersten drei Monate nach der Entlassung die vulnerabelste Phase zu sein scheint. Die Suizidrate lag hier fast 100-fach (!) über einer kürzlich ermittelten Rate in der Allgemeinbevölkerung (zitiert bei [2]). Auch fünf bis zehn Jahre nach Entlassung betrug sie immer noch fast das 30-Fache.

Weitere Verbesserungen im Entlassungsmanagement beziehungsweise eine gute Vorbereitung der Entlassung und eine intensivere Begleitung von Patienten an der Schnittstelle von stationärer zu ambulanter Betreuung in den Wochen und Monaten nach Entlassung sind unabdingbar. Dies gilt insbesondere für Patienten mit suizidalen Gedanken und Suizidversuchen bei stationärer Aufnahme, deren Risiko besonders hoch zu sein scheint. Lösungsmöglichkeiten könnten die Etablierung von Algorithmen zur Identifizierung besonders hoher Risikokonstellationen, die langsamere Ablösung von der stationären Behandlung (etwa Nutzung teilstationärer Behandlungsangebote), die Sicherstellung einer möglichst hohen Behandlerkontinuität (ambulante Weiterbehandlung durch entlassende Klinik beziehungsweise hohe Priorisierung der Zusammenarbeit mit ambulanten Therapeuten und komplementären Diensten) und niederschwellige Wiederaufnahmeangebote sein. Es liegt an uns Psychiatern, das hier aufgezeigte Problem in den Fokus zukünftiger gesundheitspolitischer Bemühungen zu rücken und Projekte zu seiner Lösung zu entwickeln.
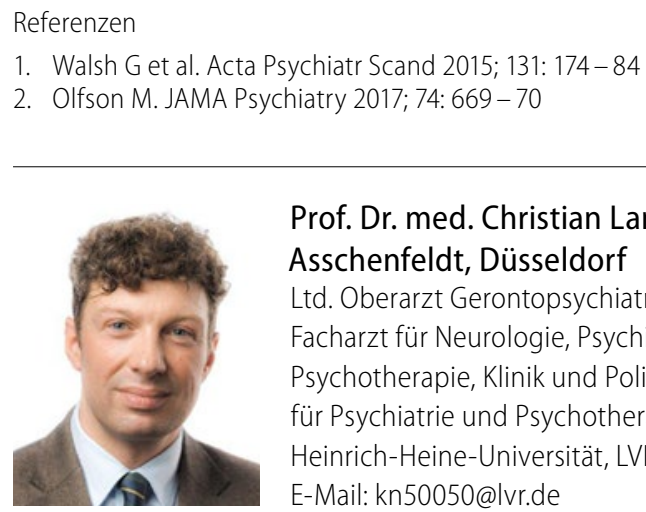

Prof. Dr. med. Christian Lange-

Asschenfeldt, Düsseldorf

Ltd. Oberarzt Gerontopsychiatrie,

Facharzt für Neurologie, Psychiatrie und

Psychotherapie, Klinik und Poliklinik

für Psychiatrie und Psychotherapie der

Heinrich-Heine-Universität, LVR-Klinikum

E-Mail:kn50050@|vr.de 